\title{
Reddening and age of six poorly studied star clusters of the Large Magellanic Cloud derived from integrated spectra ${ }^{\star}$
}

\author{
J. H. Minniti ${ }^{1}$, A. V. Ahumada ${ }^{1,2}$, J. J. Clariá ${ }^{1,2}$, and A. Benítez-Llambay ${ }^{1,2}$ \\ ${ }^{1}$ Observatorio Astronómico, Universidad Nacional de Córdoba, Laprida 854, Córdoba, CP 5000, Argentina \\ e-mail: [jminniti; andrea; claria; ale]@oac.uncor.edu \\ 2 Consejo Nacional de Investigaciones Científicas y Técnicas (CONICET), Argentina
}

Received 20 December 2013 / Accepted 24 February 2014

\section{ABSTRACT}

\begin{abstract}
Aims. To increase the number of studied star clusters (SCs) of the Large Magellanic Cloud (LMC), we present flux-calibrated integrated spectra in the optical range $(\lambda=3700-6800 \AA)$ for six poorly studied LMC SCs of IVA type. This type corresponds to the age range between 200 and $400 \mathrm{Myr}$. We also aim at creating a new template spectrum representative of this age range at the metallicity level of the LMC.

Methods. Foreground reddening $E(B-V)$ values and ages are derived by applying the template matching method that consists of comparing the line strengths and continuum distribution of the cluster spectra with those of template cluster spectra with known properties. The equivalent width (EW) of the Balmer lines and the diagnostic diagrams involving the sum of EWs of selected spectral lines were also employed as age indicators.

Results. For the first time, we provide estimates of the clusters' reddenings and ages. As expected, all the clusters appear to be of nearly the same age, their mean value being $(400 \pm 100)$ Myr, while the resulting mean $E(B-V)$ values range between 0.00 and $0.10 \mathrm{mag}$.

Conclusions. The present cluster sample complements previous ones in an effort to gather a spectral library with several clusters per age bin. By averaging the reddening-corrected integrated spectra, weighted by their signal-to-noise ratios ( $\mathrm{S} / \mathrm{N}$ ), a new high $\mathrm{S} / \mathrm{N}$ template spectrum of $400 \mathrm{Myr}$ has been created.
\end{abstract}

Key words. techniques: spectroscopic - Magellanic Clouds - galaxies: star clusters: general

\section{Introduction}

The Magellanic Clouds play an important role in interpreting distant unresolved stellar populations because high signal-tonoise ratio $(\mathrm{S} / \mathrm{N})$ integrated spectra can be obtained for their star clusters (SCs). The Large Magellanic Cloud (LMC), one of our nearest neighbours, is an irregular dwarf galaxy located at a heliocentric distance of $50 \mathrm{kpc}$ (Saha et al. 2010). Due to its proximity, their SCs can be resolved in their individual members and can be therefore studied from their colour-magnitude diagrams (CMDs). It is also possible to obtain very deep CMDs that reach even the main sequence of compact old clusters in crowded fields such as the LMC inner disc (e.g., Palma et al. 2013). Star clusters can be considered stellar population units of a given age and metallicity (Bica \& Alloin 1986a,b). This is why SC integrated spectra, as well as their templates and grids of spectral properties, have been used for stellar population synthesis studies and interpretation of galaxy spectra (e.g., Bica 1988). By comparing ages of LMC SCs derived from CMDs with those obtained from integrated spectroscopy, Asa'd et al. (2013) recently demonstrated that integrated spectra in the visible wavelength region provide very good age predictions when compared with high resolution computational models. These authors showed that integrated spectroscopy is more robust in resolving

* Integrated spectra for each star cluster are only available at the CDS via anonymous ftp to cdsarc.u-strasbg. fr (130.79.128.5) or via

http: //cdsarc.u-strasbg.fr/viz-bin/qcat?J/A+A/565/A49 the age-extinction degeneracy. To determine distances, ages and metallicities of SCs, it is necessary to first estimate the interstellar reddening affecting each cluster. As indicated by Westerlund (1997), the colour excess for an individual SC belonging to the LMC is frequently assumed to be known, adopting the value recommended in published surveys (e.g., Burstein \& Heiles 1982, hereafter BH82). However, it is important to highlight that even a small error in the $E(B-V)$ may have noticeable effects on the other quantities. Template spectra are very useful in deriving individual cluster parameters not only in our Galaxy (e.g., Schiavon et al. 2005; Ahumada et al. 2007) but also in distant galaxies (e.g., Jablonka et al. 1998; Trancho et al. 2007). They are also useful for testing stellar population models (Lyubenova et al. 2012). Integrated spectroscopy has proved to be a valuable tool to determine some fundamental properties of LMC SCs, since it provides information on reddening and age (e.g., Santos et al. 2006).

The overall recently estimated number of LMC SCs is $\approx 3100$ (Bica et al. 2008). This number, however, may be significantly larger if emission-free associations and objects related to emission nebulae are included (Bica et al. 1999). Unfortunately, however, the number of studied LMC SCs still constitutes a very small fraction of those SCs that have so far been catalogued. Therefore, research on even a handful of SCs represents an improvement in our knowledge of the LMC as a whole. The present work is part of a current project of integrated spectroscopy of concentrated SCs in the LMC that is being developed at the Observatorio Astronómico de la Universidad 
Table 1. Observed targets.

\begin{tabular}{|c|c|c|c|c|c|c|}
\hline Name $^{a}$ & $\begin{array}{c}\alpha_{2000} \\
(\mathrm{~h} \mathrm{~m} \mathrm{~s})\end{array}$ & $\begin{array}{l}\delta_{2000} \\
\left({ }^{\circ}, \prime \prime\right)\end{array}$ & $\begin{array}{c}\text { Diameter } \\
\left({ }^{\prime}\right)\end{array}$ & $\begin{array}{c}V \\
\text { (mag) }\end{array}$ & $\begin{array}{l}U-B \\
(\mathrm{mag})\end{array}$ & $\begin{array}{l}B-V \\
\text { (mag) }\end{array}$ \\
\hline NGC 1696, SL 43, ESO 56-SC04, KMHK 108 & 044830 & -681435 & 1.10 & 13.95 & 0.02 & 0.49 \\
\hline NGC 1897, SL 355, ESO 56-SC92, KMHK 722 & 051731 & -672700 & 1.05 & 13.49 & 0.18 & 0.36 \\
\hline NGC 1905, SL 369, ESO 85-SC67, KMHK 739 & 051823 & -671641 & 0.95 & 13.21 & 0.08 & 0.25 \\
\hline NGC 2166, SL 811, ESO 57-SC64, BMG 194, KMHK 1576 & 055933 & -675631 & 1.40 & 12.86 & 0.09 & 0.26 \\
\hline NGC 2181, SL 825, LW 366, ESO 86-SC54, KMHK 1602 & 060243 & -651554 & 1.50 & 13.64 & 0.07 & 0.29 \\
\hline NGC 2197, SL 838, LW 385, ESO 86-SC58, KMHK 1636 & 060607 & -670553 & 1.70 & 13.45 & 0.12 & 0.37 \\
\hline
\end{tabular}

Notes. ${ }^{(a)}$ Cluster identifications are from (SL): Shapley \& Lindsay (1963); (LW): Lyngå \& Westerlund (1963); (ESO): Lauberts (1982); (BMG): Bhatia \& MacGillivray (1989); (KMHK): Kontizas et al. (1990).

Nacional de Córdoba (Argentina) using the $2.15 \mathrm{~m}$ telescope at the Complejo Astronómico El Leoncito (CASLEO), which is located in San Juan (Argentina). This project aims to determine reddening and age for some unstudied or poorly studied SCs located in different regions of the LMC. We have already reported results based on integrated spectra for about 76 blue concentrated LMC clusters in the age range from 40 to $800 \mathrm{Myr}$ (Santos et al. 2006; Palma et al. 2008; Ahumada et al. 2009, 2011; Oddone et al. 2012; Minniti et al. 2012). Spectra of only six out of these 76 SCs were obtained with the EFOSC2 spectrograph at the New Technology Telescope (NTT) in La Silla Observatory (Chile). These studies have contributed to the individual characterisation of all these stellar systems.

The layout of this paper is as follows. The cluster sample, details about the observations and the data reduction procedure are described in Sect. 2. Section 3 deals with the determination of reddening and age through different methods. A brief discussion of the results is presented in Sect. 4, while a new template spectrum corresponding to $400 \mathrm{Myr}$ is defined in Sect. 5. The final conclusions are summarised in Sect. 6.

\section{Cluster sample and observations}

We selected six relatively populous and concentrated SCs located in the inner disc and in the outer regions of the LMC, namely: NGC 1696, NGC 1897, NGC 1905, NGC 2166, NGC 2181, and NGC 2197. As far as we know, there are no CMDs obtained and no reddening and age previous determination for these six clusters. Their compact nature and relatively high surface brightness make them good targets for integrated spectroscopy. They were selected from the catalogue of integrated $U B V$ photometry of SCs and stellar associations by Bica et al. (1996, hereafter B96), wherein 504 LMC SCs were classified in terms of their SWB types (Searle et al. 1980). These objects were chosen not only because they were not previously studied but also mainly because they are all type IVA in the sequence defined by Searle et al. (1980). According to B96, this corresponds to the 200-400 Myr age range. The observed SC sample is presented in Table 1, where cluster designations in different catalogues are provided with equatorial coordinates, angular sizes $(D)$ taken from Bica et al. (2008), integrated $V$ magnitudes, and $(U-B)$ and $(B-V)$ colours reported by B96. Figure 1 shows DSS red images $\left(5^{\prime} \times 5^{\prime}\right.$ extractions $)$ for the six selected SCs.

The observations were carried out with the "Jorge Sahade" $2.15 \mathrm{~m}$ Ritchey-Chrétien telescope at CASLEO during six nights in December 2009 and two nights in December 2012. We employed a CCD camera attached to the REOSC spectrograph (simple mode) during the two observing runs. The detector was

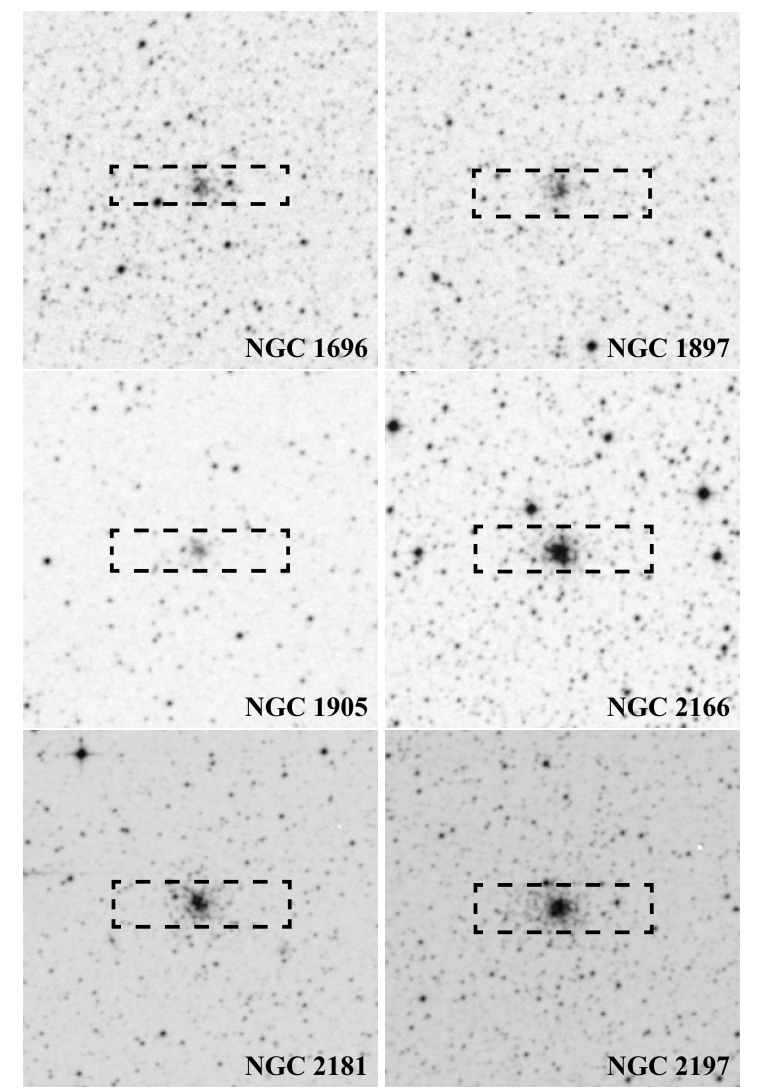

Fig. 1. DSS $5^{\prime} \times 5$ images for the studied SCs. Rectangles represent the observed regions. North is up and east is to the left.

a Tektronics chip of $1024 \times 1024$ pixels $^{2}$ of size $24 \mu \mathrm{m}$ each. One pixel corresponds to $0.94^{\prime \prime}$ on the sky. The slit was set in the East-West direction, and the observations were performed by scanning the slit across the objects in the North-South direction to get a proper sampling of the SC populations. The scanned regions are shown in Fig. 1. We used a grating of 300 grooves $/ \mathrm{mm}$, which produces an average dispersion in the observed region of $\sim 140 \AA / \mathrm{mm}(3.46 \AA / \mathrm{pix})$. The CCD operated with the usual gain and readout noise $1.98 \mathrm{e}^{-} / \mathrm{ADU}$ and $7.4 \mathrm{e}^{-}$, respectively. The useful spectral coverage was $\lambda=3700-6800 \AA$. The slit length corresponding to $4.7^{\prime}$ on the sky enabled us to sample regions of background sky. The slit width was $400 \mu \mathrm{m}\left(\sim 4.2^{\prime \prime}\right.$ in the sky), providing a resolution of $14 \AA$ as measured from the full width at half maximum (FWHM) in the $\mathrm{Cu}-\mathrm{Ar}-\mathrm{Ne}$ lines of the comparison lamps. Standard stars EG 21, LTT 1020, LTT 2415, 
Table 2. Observation log of selected clusters.

\begin{tabular}{lccc}
\hline \hline Cluster & Date & $\begin{array}{c}\text { Exposure } \\
(\mathrm{s})\end{array}$ & $\begin{array}{c}\text { Total } S / N \\
(5200-5500 \AA)\end{array}$ \\
\hline NGC 1696 & Dec. 13, 2009 & $2 \times 2700$ & 32 \\
& Dec. 14, 2009 & $2 \times 2700$ & \\
& Dec. 11, 2012 & $2 \times 3600$ & \\
NGC 1897 & Dec. 21, 2009 & $3 \times 1800$ & 31 \\
NGC 1905 & Dec. 18, 2009 & $4 \times 1800$ & 24 \\
NGC 2166 & Dec. 18, 2009 & $1 \times 1500$ & 30 \\
& & $1 \times 1800$ & \\
NGC 2181 & Dec. 19, 2009 & $2 \times 1800$ & \\
& Dec. 20, 2009 & $2 \times 1800$ & 30 \\
NGC 2197 & Dec. 21, 2009 & $2 \times 1800$ & \\
& Dec. 17, 2009 & $2 \times 1800$ & 16 \\
& Dec. 18, 2009 & $1 \times 1800$ & \\
& Dec. 14, 2012 & $1 \times 3600$ & \\
\hline
\end{tabular}

LTT 3218, and LTT 3864 from the list of Stone \& Baldwin (1983) were observed every night for flux calibrations. Bias, darks, dome, and twilight sky flat-fields were taken for reduction purposes.

To gather cluster spectra with an acceptable $\mathrm{S} / \mathrm{N}$, we obtained a series of spectra with exposure times of 30-60 min for each of the sample objects so that the total exposure time varied between 1.5 and $5 \mathrm{~h}$, depending on each cluster's brightness. Table 2 shows the log of the observations with dates, exposure times, and the resulting $\mathrm{S} / \mathrm{N}$ of the spectra.

Reductions were carried out with the Image Reduction and Analysis Facility (IRAF) ${ }^{1}$ software package, following standard procedures at the Observatorio Astronómico de la Universidad Nacional de Córdoba (Argentina). The spectra were extracted along the slit according to the cluster size and available flux. After we checked that bias and flat-fields were similar on different nights and in the two observing runs, we created a master bias and a master flat-field. The latter was constructed by taking into account both dome and sky flat-fields. The master bias was subtracted from each raw image and each scientific observation was divided by the normalised master flat-field. Since dark counts were similar to those of the bias, no dark correction was necessary to apply to the images. We subtracted the master bias and used master flat-fields to correct the frames for high and low spatial frequency variations. Background sky subtractions were then performed using pixel rows from the same frame after removing cosmic rays from the background sky regions. Precautions were taken, so that no significant background sky residuals were present on the resulting spectra. The spectral image was then converted into a one-dimensional spectrum by integrating the flux along the spatial direction of the image. Wavelength calibrations were carried out with a $\mathrm{Cu}-\mathrm{Ar}-\mathrm{Ne}$ lamp with exposures that follow the $\mathrm{SC}$ or the standard star. The rms errors involved in these calibrations are typically $0.50 \AA$ ( 0.14 pixel). Atmospheric extinction corrections, according to the site coefficients given by Minniti et al. (1989), and flux calibrations derived from the observed standard stars, were then applied. Finally, we combined individual spectra of each cluster to obtain a final spectrum with an improved $\mathrm{S} / \mathrm{N}$. The spectra were set to the rest-frame according to the Doppler shift of Balmer lines. Next, they were normalised at approximately $5800 \AA$ by avoiding spectral lines that

\footnotetext{
1 IRAF is distributed by the National Optical Astronomy Observatories, which is operated by the Association of Universities for Research in Astronomy, Inc., under contract with the National Science
} Foundation.

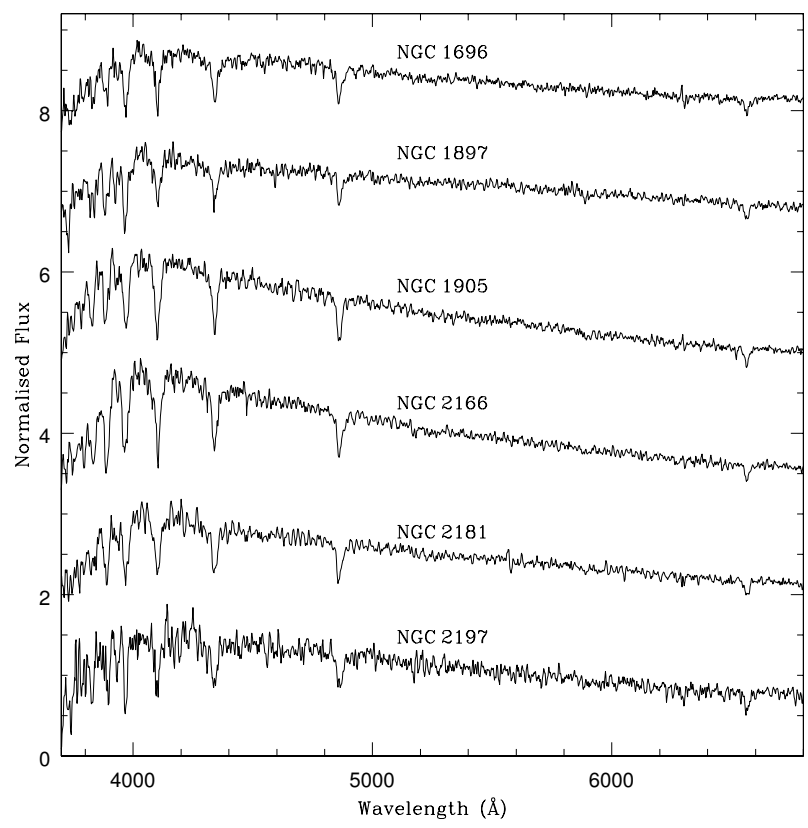

Fig. 2. Observed integrated spectra of 6 LMC clusters. The spectra are normalised at $\lambda \approx 5800 \AA$. Constants have been added to the spectra for clarity, except for the one on the bottom.

could eventually be present. While some field star contamination may be expected in the observed spectra, note that only bright field stars could affect the spectra significantly, which does not seem to occur in any of the observed clusters (see Fig. 1). Figure 2 shows the resulting flux-calibrated integrated spectra of the six observed clusters. The spectra obtained in different runs were averaged by taking into account their $\mathrm{S} / \mathrm{N}$. Constants have been added to the spectra in Fig. 2 to allow for comparison, except for the one at the bottom.

\section{Reddening and age determination}

A first estimate of the interstellar reddening affecting each cluster was made by interpolating the extinction maps of BH82. These maps were obtained from $\mathrm{HI}(21 \mathrm{~cm})$ emission data for the southern sky. They provide us with foreground $E(B-V)$ colour excesses, which depend on the Galactic coordinates. We decided not to use the full-sky maps from $100-\mu$ dust emission obtained by Schlegel et al. (1998) for the reasons given in previous papers (e.g., Geisler et al. 2003). It will be seen later that a second $E(B-V)$ estimate was obtained from the template matching method using two different templates. Columns (2)(4) of Table 3 present, for each cluster, the resulting BH82 $E(B-V)$ colour excesses, and those obtained from the template matching method after comparing the reddening corrected spectrum with one of the two used templates taken from Piatti et al. (2002, hereafter P02) spectral library, i.e., Yg (200-350 Myr) and $\mathrm{Yh}$ (500 Myr). The adopted final reddenings and ages correspond to the best matched values that are reached between the SC spectra and the different combinations of the $\mathrm{Yg}$ and $\mathrm{Yh}$ templates. Note that the finally adopted $E(B-V)$ colour excesses show a reasonable agreement with those estimated using BH82 maps. The average difference $(\Delta=$ BH82 - present value) is $\Delta E(B-V)=0.01 \pm 0.05$ (s.d.).

In a pioneering work, Bica \& Alloin (1986a, hereafter BA86a) showed that integrated spectra of small angular 
Table 3. $E(B-V)$ colour excess determinations.

\begin{tabular}{lccrr}
\hline \hline Cluster & $\begin{array}{c}E(B-V) \\
\text { BH82 }\end{array}$ & $\begin{array}{c}E(B-V) \\
\text { Yg (200-350 Myr })\end{array}$ & $\begin{array}{r}E(B-V) \\
\text { Yh }(500 \mathrm{Myr})\end{array}$ & $E(B-V)$ \\
\hline NGC 1696 & 0.04 & 0.20 & 0.05 & 0.09 \\
NGC 1897 & 0.06 & 0.20 & 0.05 & 0.09 \\
NGC 1905 & 0.06 & 0.02 & -0.15 & 0.00 \\
NGC 2166 & 0.06 & 0.02 & -0.15 & 0.00 \\
NGC 2181 & 0.03 & 0.15 & -0.03 & 0.08 \\
NGC 2197 & 0.06 & 0.15 & 0.00 & 0.10 \\
\hline
\end{tabular}

Table 4. Measurements of EWs.

\begin{tabular}{lccccccccc}
\hline \hline Name & $\begin{array}{c}\mathrm{K} \mathrm{CaII}^{1} \\
(\AA)\end{array}$ & $\begin{array}{c}\mathrm{H} \delta^{2} \\
(\AA)\end{array}$ & $\begin{array}{c}G \text { Band (CH) } \\
(\AA)\end{array}$ & $\begin{array}{c}\mathrm{H} \gamma^{4} \\
(\AA)\end{array}$ & $\begin{array}{c}\mathrm{H} \beta^{5} \\
(\AA)\end{array}$ & $\begin{array}{c}\mathrm{MgI}^{6} \\
(\AA)\end{array}$ & $\begin{array}{c}\mathrm{H} \alpha^{7} \\
(\AA)\end{array}$ & $\begin{array}{c}\mathrm{S}_{h}^{a} \\
(\AA)\end{array}$ & $\begin{array}{c}\mathrm{S}_{m}^{b} \\
(\AA)\end{array}$ \\
\hline NGC 1696 & 6.1 & 12.3 & 3.5 & 11.7 & 9.3 & 2.2 & 6.3 & 33.3 & 11.7 \\
NGC 1897 & 8.0 & 11.0 & 3.5 & 11.4 & 7.6 & 2.7 & 6.5 & 30.0 & 14.1 \\
NGC 1905 & 4.8 & 13.7 & 2.1 & 11.8 & 9.2 & 1.3 & 8.5 & 34.8 & 8.2 \\
NGC 2166 & 4.7 & 14.1 & 3.4 & 12.7 & 9.6 & 2.4 & 6.6 & 36.4 & 10.5 \\
NGC 2181 & 7.3 & 13.5 & 4.7 & 12.7 & 9.8 & 2.0 & 6.8 & 36.0 & 14.0 \\
NGC 2197 & 7.8 & 14.2 & 6.5 & 13.5 & 9.9 & 3.4 & 8.5 & 37.6 & 17.7 \\
\hline
\end{tabular}

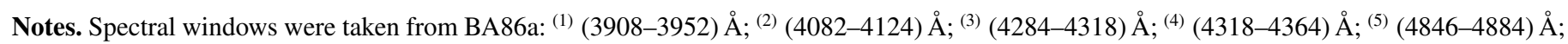
${ }^{(6)}(5156-5196) \AA$; and ${ }^{(7)}(6540-6586) \AA$. The superscript $a$ refers to $\mathrm{H} \beta, \mathrm{H} \gamma$ and $\mathrm{H} \delta$; the superscript $b$ refers to K CaII, $G$ band $(\mathrm{CH})$ and $\mathrm{MgI}$.

diameter SCs allow us to determine their basic properties, such as reddening, age, and metallicity in some cases (e.g., Ahumada et al. 2000). Thus, a direct reddening-independent age estimate was first obtained from the equivalent widths (EWs) of the Balmer absorption lines in each cluster spectrum by interpolating these values in the age calibration of Bica \& Alloin (1986b). These authors found that metallicity effects are negligible for the spectral windows that include the first four Balmer lines. More recently, using six of the same spectral windows defined in BA86a, Santos \& Piatti (2004, hereafter SP04) developed a new method to estimate $\mathrm{SCs}^{\prime}$ ages from visible integrated spectra. They defined $S_{m}$ and $S_{h}$ as the sums of the EWs of the metallic lines K CaII, $G$ band $(\mathrm{CH})$ and $\mathrm{MgI}$, and of the Balmer lines $\mathrm{H} \beta, \mathrm{H} \gamma$ and $\mathrm{H} \delta$, respectively. As shown by Dutra et al. (1999) and SP04, $S_{m}$ and $S_{h}$ prove to be useful in the discrimination of old, intermediate-age, and young systems. Also, SP04 defined diagnostic diagrams (DDs) involving $S_{h}$ and $S_{m}$ with a view to discriminating cluster ages for systems younger than 10 Gyr and metallicities for systems older than $10 \mathrm{Gyr}$.

We first defined the continuum in each spectrum according to the criteria suggested by BA86a and then we measured EWs within their selected spectral windows, using IRAF task splot. The boundaries of the spectral windows and their principal absorbers are indicated in BA86a. The EW measurements, as well as $S_{m}$ and $S_{h}$, are presented in Table 4 . Typical errors of $\sim 10 \%$ on individual EW measurements were the result of tracing slightly different continua.

We would like to point out that both Balmer line EWs and DDs of SP04 have been used only as age range indicators. The final age determination of each cluster was carried out by applying the template matching method. This procedure consists of achieving the best possible match between the analysed cluster spectrum and a template spectrum of known age and metallicity. This method presents the advantage of permitting a simultaneous determination of both foreground reddening and age (e.g., Ahumada et al. 2007; Benítez-Llambay et al. 2012). Although there exists a template library covering $\lambda<4600 \AA$ that represents the integrated spectral evolution of SCs in the Magellanic
Clouds (Santos et al. 1995), the age range of this database corresponds to clusters younger than $\sim 170 \mathrm{Myr}$, namely, those much younger than those analysed in this work. Since metallicity is not important in the spectral range we are working and/or it is only important for SCs older than 1-2 Gyr, we decided to use the solar metallicity template spectrum library created by P02. The only useful P02 template spectra for the present sample are Yg (200-350 Myr) and Yh (500 Myr), which represent moderately young stellar populations built from Galactic open clusters. To apply the template matching method, we used the software called Fast Integrated Spectra Analyzer (FISA). This software allows fast and reasonably accurate reddening and age determinations for SCs using their integrated spectra (Benítez-Llambay et al. 2012). The resulting reddening values are listed in Cols. (3)-(5) of Table 3. To perform reddening corrections, we used the normal reddening law (Seaton 1979) and the most frequently used factor 3.0 for the ratio of totalto-selective extinction (Straižys 1992). The program FISA allows us to minimise the flux residuals, which are calculated as the normalised difference (cluster - template)/cluster. The integrated spectra of the observed clusters present intermediate features between Yg and Yh P02 templates in all cases, as we show in Figs. 3, 4. Owing to these similarities, we decided to combine $\mathrm{Yg}$ and Yh templates with different weights to obtain the best possible comparison with the reddening corrected spectra. Note, however, that differences are expected to exist between cluster and template spectra due to variations in the stellar composition of the cluster, such as the presence of a relatively bright star with particular spectral features or to contamination of a field star close to the direction to the cluster. Table 5 gives in succession the first cluster designation, the age range inferred from the Balmer lines, the age range estimated from the DDs of SP04, and the ages finally determined from the template matching method.

\section{Discussion on individual clusters}

As far as we are aware, none of the observed LMC clusters has been the subject of previous detailed studies. Positions and 
J. H. Minniti et al.: Integrated spectroscopy of LMC clusters

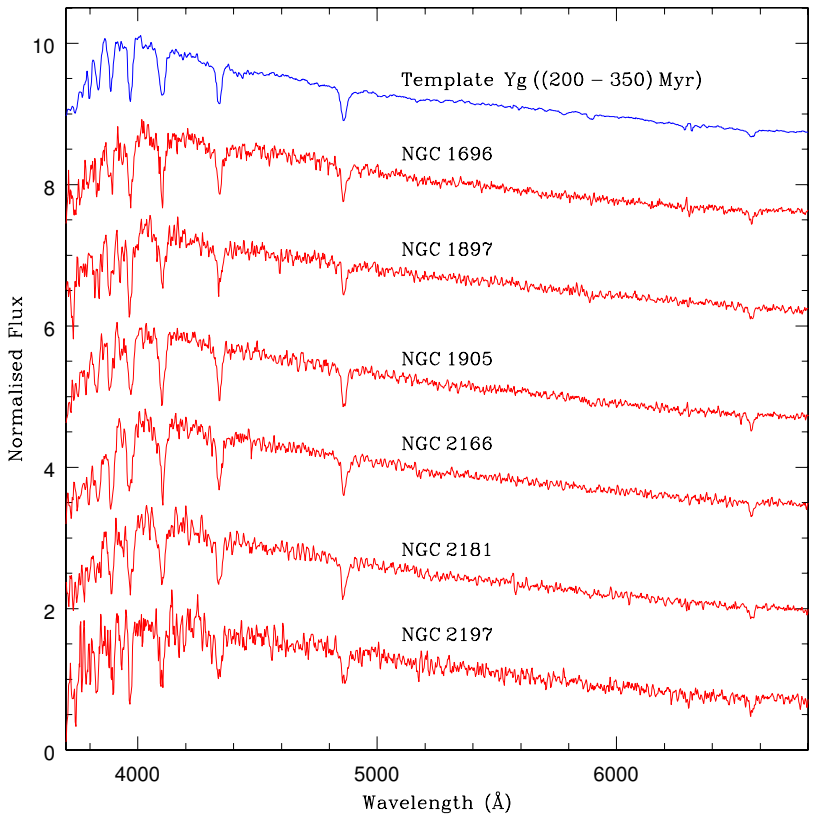

Fig. 3. Comparison of reddening-corrected spectra of the cluster sample to the Yg template (top). Units as in Fig. 2.

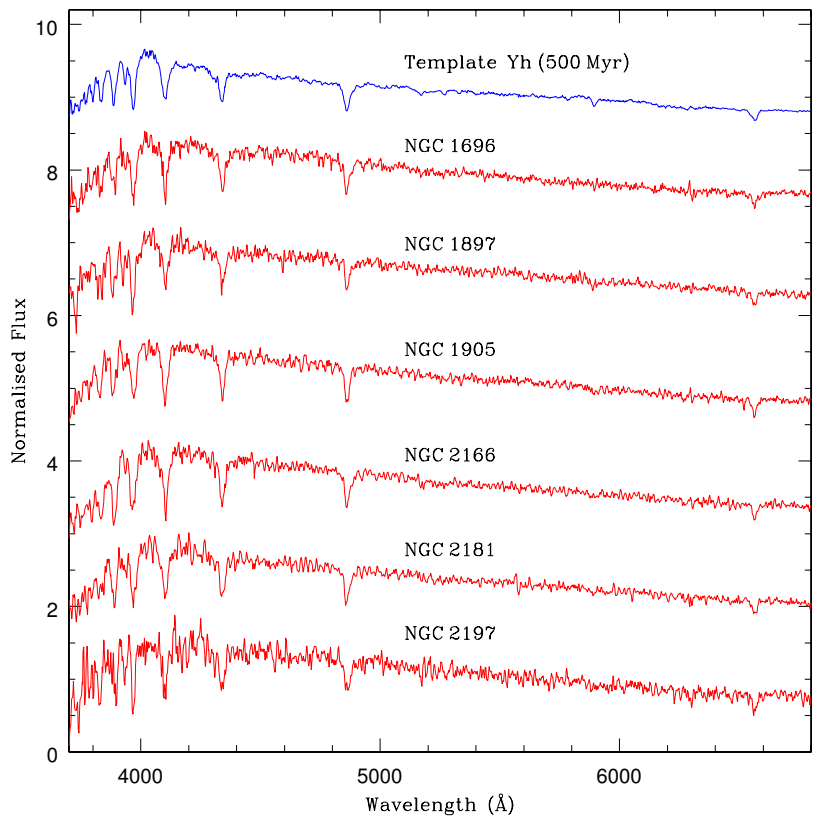

Fig. 4. Comparison of reddening-corrected spectra of the cluster sample to the Yh template (top). Units as in Fig. 2.

angular diameters were recently updated by Bica et al. (2008). Only SWB types (Searle et al. 1980) were those derived by B96 by means of integrated photometry, so the current reddening and age determinations are the first of their kind. Below, we briefly discuss the results obtained for each of the studied SCs.

\subsection{NGC 1696}

This is a SWB IVA type object whose Balmer lines suggest age values in the range between 100 and $500 \mathrm{Myr}$. On the other hand,
Table 5. Cluster ages.

\begin{tabular}{lccc}
\hline \hline Cluster & $\begin{array}{c}\text { Balmer age } \\
(\mathrm{Myr})\end{array}$ & $\begin{array}{c}\text { DD age } \\
(\mathrm{Myr})\end{array}$ & $\begin{array}{c}\text { Template age } \\
(\mathrm{Myr})\end{array}$ \\
\hline NGC 1696 & $100-500$ & $40-350 / 400-1500$ & $455 \pm 100$ \\
NGC 1897 & $100-500$ & $400-1500$ & $455 \pm 100$ \\
NGC 1905 & $100-500$ & $40-350$ & $340 \pm 100$ \\
NGC 2166 & $100-500$ & $40-350$ & $340 \pm 100$ \\
NGC 2181 & $100-500$ & $400-1500$ & $390 \pm 100$ \\
NGC 2197 & $100-500$ & $400-1500$ & $390 \pm 100$ \\
\hline
\end{tabular}

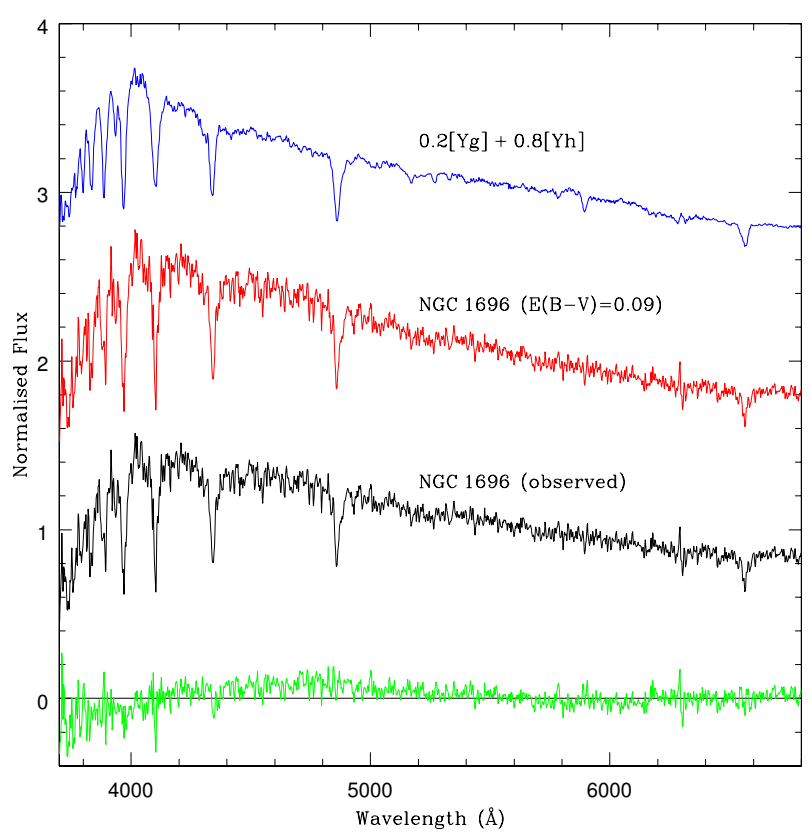

Fig. 5. Observed integrated spectrum of NGC 1696 and the spectrum corrected for $E(B-V)=0.09$ (middle), the combined template (top), and the residual flux according to $\left(F_{\text {cluster }}-F_{\text {template }}\right) / F_{\text {cluter }}$ (bottom). Units as in Fig. 2.

DDs indicate that NGC 1696 lies in the limit that corresponds to (40-350 Myr) and (400-1500 Myr) regions. As shown in Figs. 3, 4, NGC 1696 spectrum compares reasonably well for both P02 Yg (200-350 Myr) and Yh (500 Myr) templates, once the observed spectrum has been corrected by $E(B-V)=0.20$ and 0.05 mag, respectively. However, since the continuum distribution and the Balmer jump in the observed spectrum better resembles those of the older template, we decided to create an average template, where $\mathrm{Yh}$ contributes to $80 \%$ and $\mathrm{Yg}$ to $20 \%$, hence, a template with an average age of $455 \mathrm{Myr}$ (Fig. 5). After correcting the observed spectrum by $E(B-V)=0.09$, we obtained a very good match with this template. For NGC 1696 and the other clusters, $E(B-V)$ was determined with a typical uncertainty between 0.01 and $0.03 \mathrm{mag}$, which represents the minimum reddening variation needed to distinguish the observed spectrum from the template one.

\subsection{NGC 1897}

Likewise, NGC 1897 presents no previous reddening or age determinations. From B96 we barely know that NGC 1897 also belongs to the SWB IVA type. Although the comparisons of the observed spectrum with Yg and Yh templates are good enough and their corresponding residual fluxes are small, we note the 


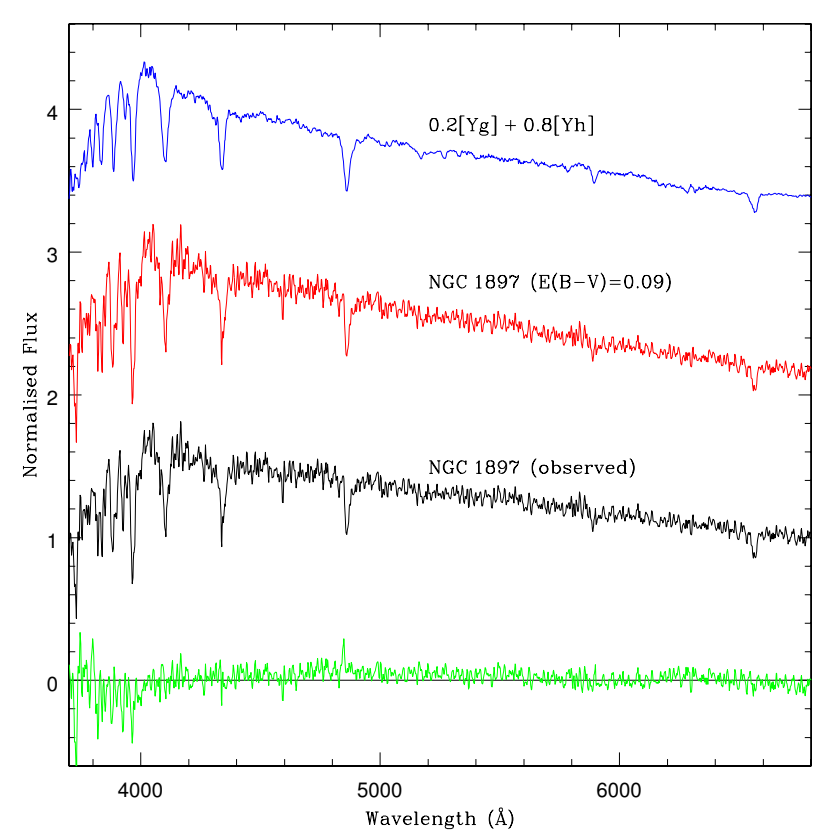

Fig. 6. Observed integrated spectrum of NGC 1897 and the spectrum corrected for $E(B-V)=0.09$ (middle), the combined template (top), and the residual flux according to $\left(F_{\text {cluster }}-F_{\text {template }}\right) / F_{\text {cluter }}$ (bottom). Units as in Fig. 2.

comparison with $\mathrm{Yh}$ (Fig. 4) is slightly better than that of $\mathrm{Yg}$ (Fig. 3), which is basically due to the intensity of the K CaII line $(\lambda \approx 3933 \AA)$ in the observed spectrum. The inferred reddenings in both cases are $E(B-V)=0.20$ and 0.05 mag, respectively. After several combinations of $\mathrm{Yg}$ and $\mathrm{Yh}$ templates, the best match turned out to be with the same template built for NGC 1696, once the observed spectrum was corrected by $E(B-V)=0.09$ (Fig. 6).

\subsection{NGC 1905}

As in the above cases, NGC 1905 is an interesting cluster because it has no previous reddenning and age determinations. The Balmer lines suggest an age of $\sim 300 \mathrm{Myr}$, which is consistent with the suggested by the DDs (40-350 Myr). The integrated spectrum of NGC 1905 is quite similar to the $\mathrm{Yg}$ and Yh templates, if it is previously corrected by $E(B-V)=0.02$ and -0.15 mag (Figs. 3, 4), respectively. Since the continuum distribution, the Balmer jump, the intensity of the $\mathrm{K} \mathrm{CaII} \mathrm{line} \mathrm{and}$ the intensity of the Balmer lines seem to favour the younger template, a combined template in which Yg contributes to $70 \%$ and Yh to $30 \%$ yielded the best match with the observed spectrum (Fig. 7). Therefore, we adopted a colour excess $E(B-V)=0.00$ and an age of $(340 \pm 100)$ Myr for NGC 1905.

\subsection{NGC2166}

According to B96, NGC 2166 is also a SWB IVA type cluster. Both Balmer lines and DDs suggest that the age of NGC 2166 should range between 40 and $500 \mathrm{Myr}$. The spectral features of this cluster are remarkably similar to those of $\mathrm{Yg}$ and $\mathrm{Yh}$ templates, provided that the observed spectrum is corrected beforehand by $E(B-V)=0.02$ and -0.15 mag, respectively (Figs. 3, 4). The continuum distribution and the Balmer jump, however, seem

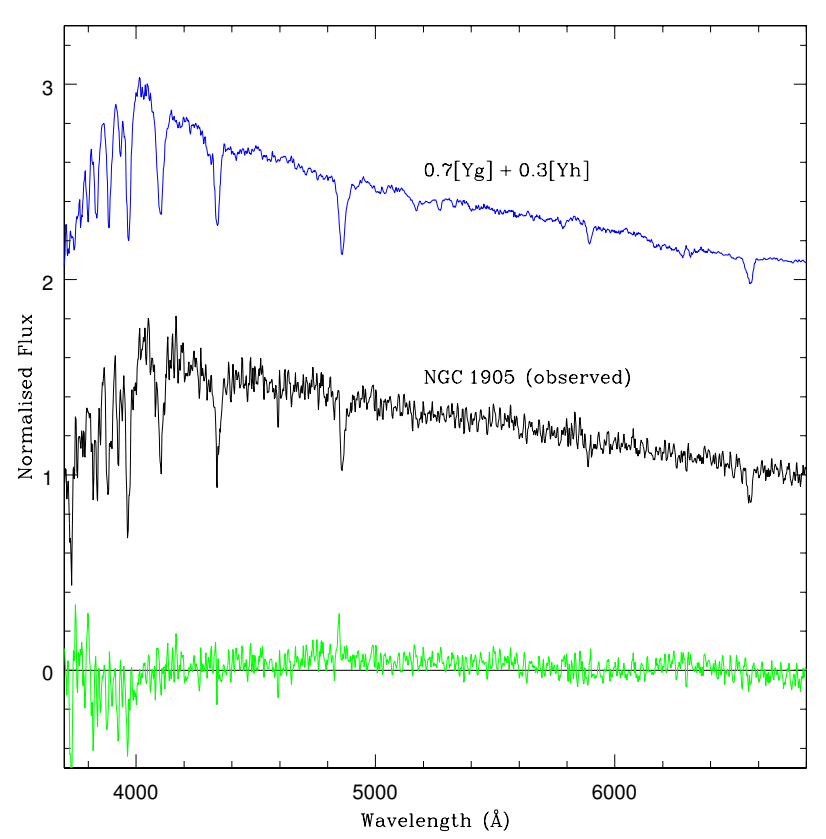

Fig. 7. Observed integrated spectrum of NGC 1905 (middle), the combined template (top), and the residual flux according to ( $F_{\text {cluster }}-$ $\left.F_{\text {template }}\right) / F_{\text {cluter }}$ (bottom). Units as in Fig. 2.

to favour the template $\mathrm{Yg}$. For this reason, we used the same combination of Yg and Yh templates as for NGC 1905 (Fig. 8). We conclude that NGC 2166 is not affected by reddening and we adopt an age of $(340 \pm 100) \mathrm{Myr}$, according to the weights assigned to each of the templates.

\subsection{NGC 2181}

Like the other clusters, NGC 2181 is a SWB IVA type object which has not been studied yet. The Balmer lines suggest age values between 100 and $500 \mathrm{Myr}$, while the DDs indicate that this cluster falls within the (400-1500) Myr region. As shown in Figs. 3, 4, a reasonable match is found when the cluster spectrum is compared with both $\mathrm{Yg}$ and $\mathrm{Yh}$ templates, once the cluster spectrum has been corrected for $E(B-V)=0.15$ and -0.03 mag, respectively. When it is compared with the Yg template (Fig. 3), an excellent agreement in the continuum distribution for $\lambda>$ $4000 \AA$ is seen. The main lines in this spectral region appear in both NGC 2181 and in the Yg template. The comparison in the region of the $4000 \AA$ break is, however, not so good. On the other hand, when the cluster spectrum is compared to the $\mathrm{Yh}$ template (Fig. 4), a better comparison towards $\lambda<4000 \AA$ is observed. As we show in Fig. 9, the best match corresponds to a template where $\mathrm{Yg}$ and $\mathrm{Yh}$ contribute in the same way at $50 \%$ each, adopting $E(B-V)=0.08$ and an intermediate age between both templates $(390 \pm 100 \mathrm{Myr})$.

\subsection{NGC 2197}

NGC 2197 is a compact group of stars also classified by B96 as belonging to SWB IVA type. Its low surface brightness explains the comparatively low $\mathrm{S} / \mathrm{N}$ of the integrated spectrum (Table 2). As in the other cases, the Balmer lines suggest age values between 100 and $500 \mathrm{Myr}$, while the DDs indicate that this cluster falls within the (400-1500) Myr region. In spite of the low S/N, 


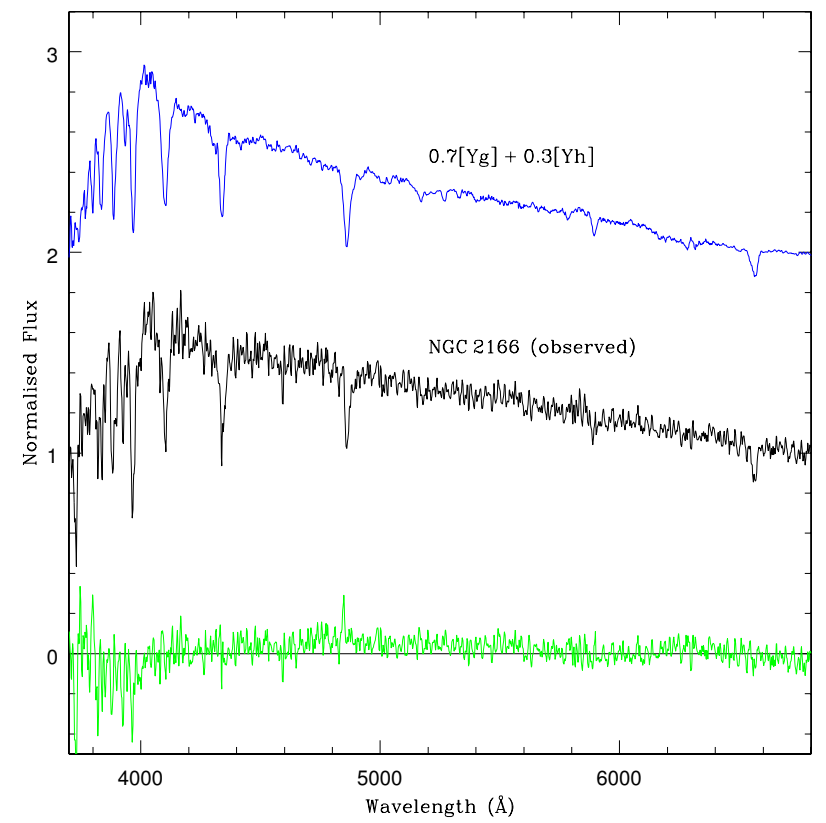

Fig. 8. Observed integrated spectrum of NGC 2166 (middle), the combined template (top), and the residual flux according to ( $F_{\text {cluster }}-$ $\left.F_{\text {template }}\right) / F_{\text {cluter }}$ (bottom). Units as in Fig. 2.

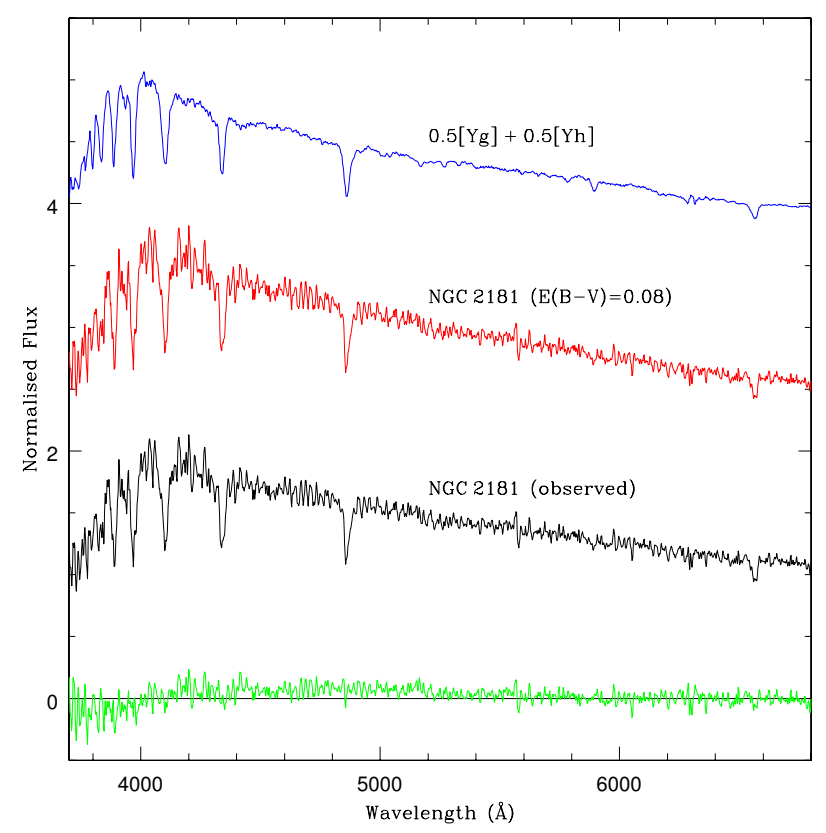

Fig. 9. Observed integrated spectrum of NGC 2181 and the spectrum corrected for $E(B-V)=0.08$ (middle), the combined template (top), and the residual flux according to $\left(F_{\text {cluster }}-F_{\text {template }}\right) / F_{\text {cluter }}$ (bottom). Units as in Fig. 2.

it can be seen that the NGC 2197 spectrum compares reasonably well with Yg and Yh templates in Figs. 3, 4, once the observed spectrum has been corrected by $E(B-V)=0.15$ and 0.0 mag, respectively. It is very probable that the age of NGC 2197 is very close to that age in which the Balmer lines reach their maximum intensity. Therefore, the best match is obtained by making the

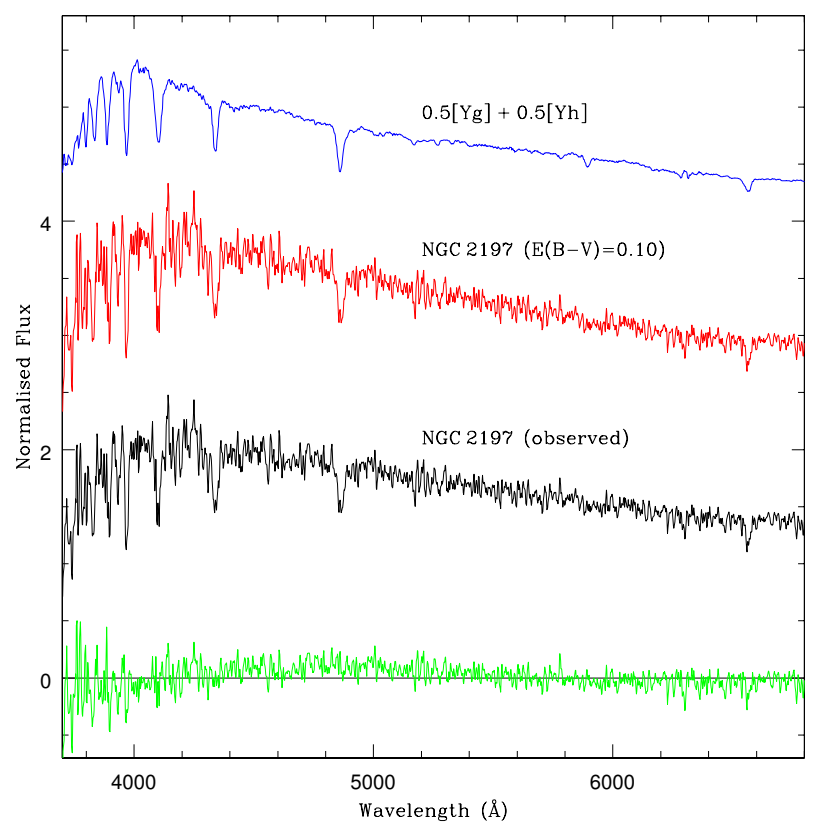

Fig. 10. Observed integrated spectrum of NGC 2197 and the spectrum corrected for $E(B-V)=0.10$ (middle), the combined template (top), and the residual flux according to $\left(F_{\text {cluster }}-F_{\text {template }}\right) / F_{\text {cluter }}$ (bottom). Units as in Fig. 2.

same combination created for NGC 2181 after correcting the observed spectrum by $E(B-V)=0.10$ (Fig. 10).

\section{New template spectrum}

As shown in Figs. 3, 4, the six studied clusters present intermediate features between those of the Yg and Yh templates defined by P02. Such similarities are seen not only in the spectral continuum distribution but also in the presence and intensity of the quite similar absorption lines. Considering the ages finally adopted for the individual clusters (Table 5), we decided to assume an age value of $(400 \pm 100)$ Myr for the whole sample. Although the $\mathrm{S} / \mathrm{N}$ of the individual observed spectra may be considered acceptable, we averaged these spectra weighting them by their $\mathrm{S} / \mathrm{N}$ to define a new Ygh template spectrum with a high $\mathrm{S} / \mathrm{N}$. Such a template improves the temporal resolution of the P02 spectral library because it represents the integrated properties of SCs that are aged at approximately 400 Myr. Before combining the individual spectra, they were reddening-corrected, according to the adopted $E(B-V)$ values (Table 3, Col. 5). The new defined template Ygh is shown in Fig. 11 (top) with the reddening-corrected individual spectra of the six SCs used to create it.

\section{Concluding remarks}

As part of a systematic spectroscopic survey of SCs in the LMC, we present and analyse flux-calibrated integrated spectra in the optical range for six concentrated SCs located within the inner disc and in the outer regions of the LMC. None of these SCs were the subject of previous detailed studies. For the first time we derive here both foreground $E(B-V)$ reddening values and ages. The derived $E(B-V)$ colour excesses are in the range of $0.00 \leq E(B-V) \leq 0.10$ (Table 3). Such variations in Galactic 


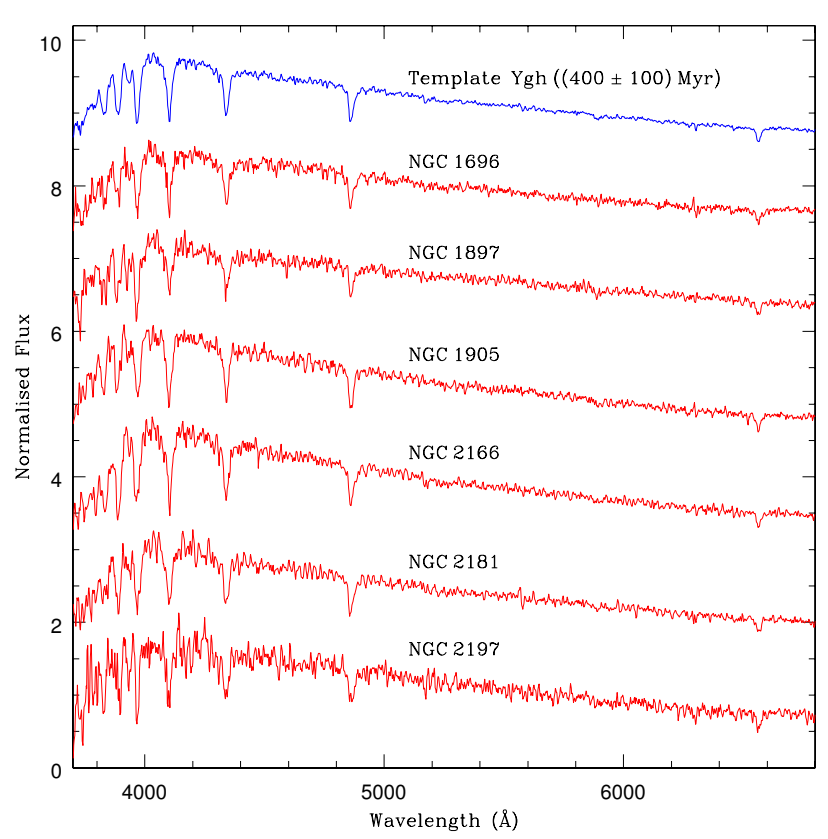

Fig. 11. New Ygh template spectrum for the $(400 \pm 100)$ Myr age range and the reddening-corrected cluster spectra used for its construction. Units as in Fig. 2.

reddenings are to be expected because the distribution of the present clusters covers as much as $\sim 19^{\circ}$ on the sky. Since the six clusters are found to be nearly the same age, a new template has now been created. The Ygh template is representative of ages around $400 \mathrm{Myr}$ at the metallicity level of the LMC. This enables us to improve the existing cluster spectral libraries. The present work provides us with a further step in stellar population synthesis studies in galaxies.

Acknowledgements. We thank the warm hospitality and assistance of CASLEO staff during the observations. We thank the referee, Francisco Maia, for helping us to improve this manuscript. This work was supported by the Argentinian institutions CONICET, Agencia Nacional de Promoción Científica y Tecnológica and SECYT (Universidad Nacional de Córdoba). This research has made use of NASA's Astrophysics Data System, Digitized Sky Survey and of the SIMBAD database, operated at CDS, Strasbourg, France. The Digitized Sky Surveys were produced at the Space Telescope Science Institute under US Government grant NAG W-2166. The images of these surveys are based on photographic data obtained using the Oschin Schmidt Telescope on Palomar Mountain and the UK Schmidt Telescope. The plates were processed into the present compressed digital form with the permission of these institutions.

\section{References}

Ahumada, A. V., Clariá, J. J., Bica, E., \& Piatti, A. E. 2000, A\&AS, 141, 79 Ahumada, A. V., Clariá, J. J., \& Bica, E. 2007, A\&A, 473, 437

Ahumada, A. V., Talavera, M. L., Clariá, J. J., et al. 2009, in IAU Symp. 256 , eds. J. T. Van Loon, \& J. M. Oliveira, 293

Ahumada, A. V., Benítez-Llambay, A., Santos, Jr., J. F. C., et al. 2011, Bol. Asoc. Arg. Astron., 54, 171

Asa'd, R. S., Hanson, M. M., \& Ahumada, A. V. 2013, PASP, 125, 1304

Benítez-Llambay, A., Clariá, J. J., \& Piatti, A. E. 2012, PASP, 124, 173

Bhatia, R. K., \& MacGillivray, H. T. 1989, A\&A, 211, 9

Bica, E. 1988, A\&A, 195, 76

Bica, E., \& Alloin, D. 1986a, A\&A, 162, 21

Bica, E., \& Alloin, D. 1986b, A\&AS, 66, 171

Bica, E., Clariá, J. J., Dottori, H., Santos, Jr., J. F. C., \& Piatti, A. E. 1996, ApJS, 102,57

Bica, E. L. D., Schmitt, H. R., Dutra, C. M., \& Oliveira, H. L. 1999, AJ, 117, 238

Bica, E., Bonatto, C., Dutra, C. M., \& Santos, J. F. C. 2008, MNRAS, 389, 678

Burstein, D., \& Heiles, C. 1982, AJ, 87, 1165

Dutra, C. M., Bica, E., Clariá, J. J., \& Piatti, A. E. 1999, MNRAS, 305, 373

Geisler, D., Piatti, A. E., Bica, E., \& Clariá, J. J. 2003, MNRAS, 341, 771

Jablonka, P., Bica, E., Bonatto, C., et al. 1998, A\&A, 335, 867

Kontizas, M., Morgan, D. H., Hatzidimitriou, D., \& Kontizas, E. 1990, A\&AS, 84,527

Lauberts, A. 1982, ESO/Uppsala survey of the ESO(B) atlas

Lyngå, G., \& Westerlund, B. E. 1963, MNRAS, 127, 31

Lyubenova, M., Kuntschner, H., Rejkuba, M., et al. 2012, A\&A, 543, A75

Minniti, D., Clariá, J. J., \& Gómez, M. N. 1989, Ap\&SS, 158, 9

Minniti, J. H., Ahumada, A. V., Clariá, J. J., et al. 2012, Bol. Asoc. Arg. Astron., 55,119

Oddone, M. A., Palma, T., Ahumada, A. V., et al. 2012, Bol. Asoc. Arg. Astron., 55,131

Palma, T., Ahumada, A. V., Clariá, J. J., Santos, Jr., J. F. C., \& Bica, E. 2008, Acta Astron., 58, 359

Palma, T., Clariá, J. J., Geisler, D., Piatti, A. E., \& Ahumada, A. V. 2013, A\&A, 555, A131

Piatti, A. E., Bica, E., Clariá, J. J., Santos, J. F. C., \& Ahumada, A. V. 2002, MNRAS, 335, 233

Saha, A., Olszewski, E. W., Brondel, B., et al. 2010, AJ, 140, 1719

Santos, Jr., J. F. C., \& Piatti, A. E. 2004, A\&A, 428, 79

Santos, Jr., J. F. C., Bica, E., Clariá, J. J., et al. 1995, MNRAS, 276, 1155

Santos, Jr., J. F. C., Clariá, J. J., Ahumada, A. V., et al. 2006, A\&A, 448, 1023

Schiavon, R. P., Rose, J. A., Courteau, S., \& MacArthur, L. A. 2005, ApJS, 160, 163

Schlegel, D. J., Finkbeiner, D. P., \& Davis, M. 1998, ApJ, 500, 525

Searle, L., Wilkinson, A., \& Bagnuolo, W. G. 1980, ApJ, 239, 803

Seaton, M. J. 1979, MNRAS, 187, 73P

Shapley, H., \& Lindsay, E. M. 1963, Irish Astron. J., 6, 74

Stone, R. P. S. \& Baldwin, J. A. 1983, MNRAS, 204, 347

Straižys, V. 1992, Multicolor Stellar Photometry (Pachart Pub. House)

Trancho, G., Bastian, N., Miller, B. W., \& Schweizer, F. 2007, ApJ, 664 284

Westerlund, B. E. 1997, The Magellanic Clouds (Cambridge Univ. Press), Cambridge Astrophys. Ser., 29 